\title{
Foreword by Guest Editors for the Special Issue on the 2012 International Conference on Mobility for Life: Technology, Telecommunication and Problem Based Learning (TTPBL) Conference
}

\author{
Bhawani Shankar Chowdhry • \\ Sandeep Shrinivas Inamdar • Romyen Kosaikanont
}

Published online: 26 February 2013

(C) Springer Science+Business Media New York 2013

Welcome to this special issue of Wireless Personal Communications. This special issue comprises a selection of best papers from the First International Conference on Mobility for Life: Technology, Telecommunication and Problem Based Learning (TTPBL) Conference. This three days event provided a dynamic platform for experts, scholars, higher education policy makers and private sector parties from Europe, Asia and other parts of the world to exchange ideas, research findings, innovations, best practices, lesson learned and challenges encountered throughout the research process, as well as the implementation of technology, telecommunication and problem-based learning. In order to share an understanding of problem-based learning both in theory and practice, with higher education personnel from Europe and Asia, particularly participating in the Erasmus Mundus Project and implementing PBL in their institutions, an exchange of innovative knowledge and research findings in the fields of technology and telecommunications were shared at threadbare level. In addition, this event aided in deriving information on good practices and knowledge on PBL for dissemination to the academic community as well as to the private sector and helped in generating a network of scholars in the fields of PBL, technology and telecommunication.

All these papers have been extended and reviewed again by three independent reviewers. The TTPBL Conference (www.pbltt2011.mfu.ac.th) jointly organized by Mae Fah Luang University and Aalborg University, Denmark together with the consortium of 18 universities from Europe and Asia under the Erasmus Mundus Mobility Programme at Mae Fah Luang

\footnotetext{
B. S. Chowdhry $(\bowtie)$

Faculty of Electrical, Electronics, Telecommunication and Computer Engineering, Mehran University of Engineering and Technology, Jamshoro, Sindh 76062, Pakistan

e-mail: c.bhawani@ieee.org

S. S. Inamdar

Vishwa-Niketan/Centre for Telecommunication Infrastructure (CTIF-India),

Kharghar, Navi Mumbai 410210, India

e-mail: ssinamdar31@rediffmail.com; jangurukul@gmail.com

R. Kosaikanont

Economics, School of Management, Mae Fah Luang University, Muang, Chiang Rai 57100, Thailand e-mail: romyen@mfu.ac.th
} 
University, Chiang Rai during the 5-7th March 2012. Twenty three papers on the conference theme: Technology, Telecommunication and Problem Based Learning presented out of almost 70 abstracts submission. However, only nine best papers are selected for this special issue.

The first paper entitles "Optimal Design of Remote Terminal Unit (RTU) for Wireless SCADA System for Energy Management”. The design of RTU using FPGA resulted in optimized solution to cater energy management applications. Traditional RTUs have limitation for implementing complex scenarios like energy management using wireless SCADA. Therefore FPGA based reliable and reconfigurable RTUs are preferred over conventional systems. In addition to optimized hardware design using FPGA, this paper also focused on optimization of wireless link associated with RTU.

The second paper entitles "Successful Transformation of ICT Graduate Program: A Role Model for Developing Countries". This paper presents a real case study in which curriculum of postgraduate was set in away to generate huge interest, utilize expertise and resources of various departments under one umbrella, creation of inspiring atmosphere to attract good number of post graduate students. The transformation was successfully done at Institute of Information and Communication Technologies in Mehran University of Engineering and Technology, Jamshoro, Pakistan.

The third paper entitles "Mobile Technology: Opportunity for Entrepreneurship". This paper reviews the recent technology trends in mobile communication and mobile application market trends. The opportunity for new entrepreneurs is also identified by describing the promotion measures by Mobile Technology for Thailand (MT2), a group of government, industry and academic institutes formed to promote mobile application development in Thailand.

The fourth paper entitles "RCM: A User Friendly Router Configuration Machine". RCM is user-friendly, easy implementable, mistake immune in configuration of all types of Cisco Routers. RCM also provides network topology monitoring and updating. Thus, RCM will be the next generation method of configuration of routers and annihilating the monopoly of CCNA experts in the industry.

The fifth paper entitles "Distribution of $3 \mathrm{G}$ services among rural towns: case study of Bhutan". The distribution of $3 \mathrm{G}$ services among rural towns are modeled and optimized by the famous linear programming. The proposed model involves both population coverage and expected income, which can be easily utilized by state telecommunication enterprise of any developing country. The example is made based on the case of three border towns of Bhutan.

The sixth paper entitles "Factors Affecting Consumer Acceptance Mobile Broadband Services with Add-on Advertising: Thailand Case Study". This research attempted to determine factors that influence Thai consumer's intention to accept mobile broadband services with add-on advertising. The extended model from Theory of Reasoned Action (TRA) is used for testing user acceptance on selected groups of mobile broadband consumers in Thailand.

The seventh paper entitles "Effect of Culture, Age, and Language on Quality of Services and Adoption of IP Applications". This study proposed factors that can be used as dimensions for assessment of perception of quality of service (QOS) of IP application by users with different human centric factors. The proposed Perceived QOS comes from three macro dimensions; Cultural Dimensions, Ageing Dimensions, and Language Dimensions. With the proposed perceived QOS, application developers can identify features and quality required by users with different culture, ageing and language.

The eighth paper entitles "The First Year Nursing Students' Achievement and Critical Thinking in Local Wisdom Course Using Problem Based Learning Process". The objective of this one group - pretest and posttest design classroom research was to examine learning achievement, critical thinking and satisfaction of first year nurse students at school of nursing 
during academic year 2011. The important outcome of PBL process is provided students to be lifelong learners.

The ninth paper entitles "Energy Efficient Four Level Cooperative Opportunistic Communication for Wireless Personal Area Networks (WPAN)". This paper proposes a new cooperative opportunistic four level cooperative model for IEEE 802.15.4 Wireless Personal Area Network (WPAN). After increasing the cooperation levels correspondingly, significant energy efficiency is achieved which is a promising result.

The final paper entitles "Problem Based Learning (PBL) For Engineering Education in India: Need and Recommendations". This paper gives an overview of Indian higher education landscape and scope of PBL to Indian engineering education. It reveals that there is an urgent need to build model institute based on PBL philosophy for sustainable growth of PBL in India.

We would like to thank the Editor-in-chief (Professor Ramjee Prasad) and Springer's senior editorial assistant (Christine Velarde) for their support and help in realizing this special issue. Special thanks to all the authors for promptly revising their papers to meet the requirements of reviewers. Thanks to the organizers of TTPBL conference, especially to Ms. Nuya Leewanich, Head, Division of International Affairs, Mae Fah Luang University for coordinating with all authors to meet time lines. Hope you will have the benefit by reading this special issue.

\section{Author Biographies}

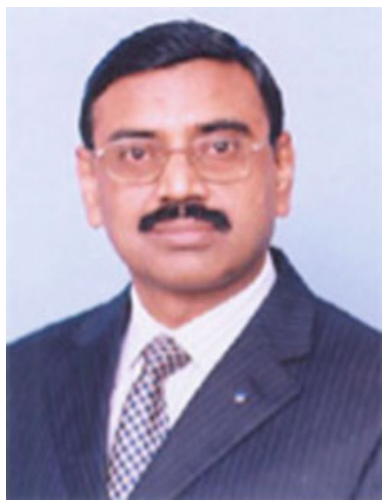

Bhawani Shankar Chowdhry is the Dean Faculty of Electrical, Electronics, Telecommunication and Computer Engineering at Mehran University of Engineering and Technology, Jamshoro (MUET), Pakistan. He did his B.E. in 1983 from MUET and Ph.D. in 1990 from School of ECS, University of Southampton, UK. He has 30 years of teaching, research and administrative experience in the field of Information and Communication Technology. He has the honour of becoming one of the editor of books "Wireless Networks, Information Processing and Systems", CCIS 20, and "Emerging Trends and Applications in Information Communication Technologies", CCIS 281, published by Springer Verlag, Germany. His list of research publication crosses to over 60 in national and international journals, IEEE and ACM proceedings. Also, he has Chaired Technical Sessions in USA, UK, China, UAE, Italy, Sweden, Finland, Switzerland, Pakistan, Denmark, and Belgium. He is member of various professional bodies including: Chairman IEEE Communication Society (COMSOC), Karachi Chapter, Region10 Asia/Pacific, Fellow IEP, Fellow IEEEP, Senior Member, IEEE Inc. (USA), Senior Member ACM Inc. (USA). 


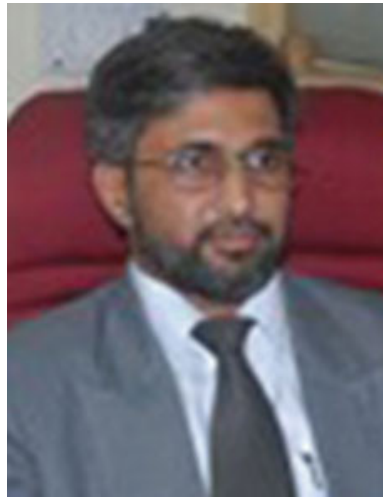

Sandeep Shrinivas Inamdar is bachelor in Electrical Engineering and completed Masters Degree in control systems. He completed his Doctoral programme from NITIE in Electrical Distribution System Efficiency Improvement. He is currently secretary of Vishwa-Niketan, co director, CTIF India, trustee, GISFI (www.gisfi.org.in) and was Director of Sinhgad Technical Education Society, Lonavala campus and Principal of Sinhgad Institute of Technology. He has total 25 years experience in teaching out of which 17 years of leading academic campuses in capacity of Director and Principal at Pune, Mumbai and Delhi. He is also member of Department of Science and Technology (NCSTC Division) Government of India, Expert Committee. He has participated in two European Commission Projects so far and is currently contributing as Steering Committee Member of Erasmus Mundus program of European Commission titled "Mobility for Life". He is a recognized Ph.D. guide in project based learning with UNESCO chair in Aalborg University Denmark. He has travelled across the globe and has collaborative programs with few well known universities in the world. He has organized more than 15 International Workshops and conferences. He is deeply interested in Problem Based Learning activities involving user industry and evolved more than 15 skill development programs for students in India.

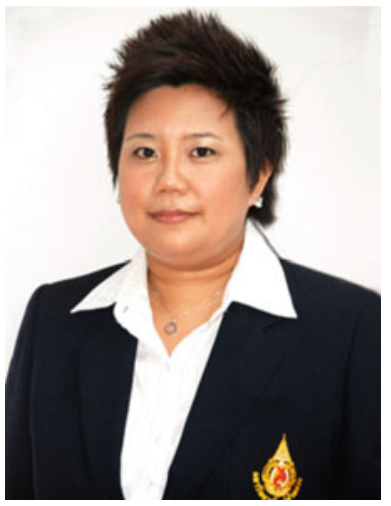

Romyen Kosaikanont is an Assistant to the President of Mae Fah Luang University and also a lecturer in Economics, School of Management. By training, she is a feminist economist and received her Master Degree in Gender and Development from Institute of Development Studies, University of Sussex and Ph.D. in Economics and International Development, University of Bath, UK. Her main research interests and community engagement works are on Gender and Urban Environmental Management in South East Asia (funded by CIDA-AIT), Participatory Urban Community Development (Thai Foundation), and Informal Female Workers Rights (OXFAM SADA). Her recent action research is on Community Archeology employing participatory approach in conducting archeological and historical research in Mae Hong Son Province. 